\title{
Management of low back pain: knowledge and adherence to clinical practice guidelines amongst physiotherapists in selected hospitals in lagos state
}

\author{
Akodu AK, Kareem RO, Faniyi OR.
}

\begin{abstract}
Objective: The therapeutic management of patients with Low Back Pain (LBP) has long been characterized by considerable variation among physiotherapists within and between countries. Over the past decade, systematic reviews have been written, and clinical practice guidelines have been made available. However, knowledge and adherence to clinical practice guidelines seem to be challenging as developing them in the first place. This study was therefore aimed at determining the knowledge and adherence to LBP clinical practice guidelines amongst Physiotherapist in selected hospitals in Lagos State.
\end{abstract}

Methods: A total of 154 practicing physiotherapists based in Lagos State participated in this study. They were required to complete a 34-item questionnaire which collected information on demographic data, work experience, treatment activities and their knowledge and adherence to the LBP clinical practice guidelines.

Results: Only a small percentage $(28.30 \%)$ of the respondents is knowledgeable while even a smaller population $(21.70 \%)$ adhered to the LBP clinical practice guideline. Knowledge and adherence to LBP clinical practice guidelines were influenced by setting of practice $(\mathrm{p}=0.05, \mathrm{p}=0.01)$ and area of specialization $(\mathrm{p}=0.02, \mathrm{p}=0.01)$ of the physiotherapists. However, age $(\mathrm{p}=0.70, \mathrm{p}=0.13)$ and involvement in the management of LBP $(\mathrm{p}=0.23, \mathrm{p}=0.35)$ did not influence knowledge and adherence to LBP clinical practice guidelines.

Conclusion: A small population of the respondents are knowledgeable and adhere to the LBP clinical practice guidelines and some of the characteristics of the physiotherapists influence the knowledge and adherence to LBP clinical practice guidelines.

Keywords: Low back pain, Clinical practice Guidelines, Knowledge, Adherence

Correspondence author:Akodu AK.,Email: aakodu@unilag.edu.ng,akoduashiyat@ gmail.com

"Department of physiotherapy, College of Medicine, University of Lagos, Lagos Nigeria

Research Journal of Health Sciences subscribed to terms and conditions of Open Access publication. Articles are distributed under the terms of Creative Commons Licence (CC B Y-NC-ND 4.0). (http://creativecommons.org/licences/by-nc-nd/4.0).

http://dx.doi.org/10.4314/wsa.v4i3.4 


\title{
Gestion de la lombalgie: la connaissance et le respect des directives de pratique clinique parmi les physiothérapeutes dans les hôpitaux sélectionnés dans l'état lagos
}

\author{
Akodu AK, Kareem RO, Faniyi OR.
}

\begin{abstract}
Resume
Objectif: La prise en charge thérapeutique des patients atteints de lombalgie (LBP) a longtemps été caractérisée par des écarts considérables entre les physiothérapeutes au sein et entre les pays. Au cours de la dernière décennie, les examens systématiques ont été écrits, et les lignes directrices de pratique clinique ont été mis à disposition. Cependant, la connaissance et le respect des directives de pratique clinique semblent être difficile car les développer en premier lieu. Cette étude a donc pour but de déterminer la connaissance et le respect des LBP lignes directrices de pratique clinique parmi Physiothérapeute dans les hôpitaux sélectionnés dans l'Etat de Lagos.
\end{abstract}

Méthodes: Un total de 154 physiothérapeutes pratiquant basé à Lagos State a participé à cette étude. Ils étaient tenus de remplir un questionnaire de 34 items qui a recueilli des informations sur les données démographiques, l'expérience de travail, les activités de traitement et de leur connaissance et le respect des lignes directrices de pratique clinique LBP.

Résultats: Seul un faible pourcentage (28,30\%) des répondants connaît alors même une population plus petite $(21,70 \%$ de) a adhéré à la LBP guide de pratique clinique. La connaissance et le respect des LBP lignes directrices de pratique clinique ont été influencés par la mise en pratique $(p=0,05, p=0,01)$ et domaine de spécialisation ( $\mathrm{p}=0,02, \mathrm{p}=0,01)$ des physiothérapeutes. Cependant, l'âge $(\mathrm{p}=0,70, \mathrm{p}=0,13)$ et l'implication dans la gestion de la lombalgie $(p=0,23, p=0,35)$ n'a pas influé sur la connaissance et le respect des LBP lignes directrices de pratique clinique.

Conclusion: Une petite population des répondants sont informés et respecte les lignes directrices de pratique clinique LBP et certaines des caractéristiques des physiothérapeutes influencent la connaissance et le respect des LBP lignes directrices de pratique clinique.

Mots-clés: lombalgie, Lignes directrices de pratique clinique, la connaissance, le respect

Auteur correspondant:.A.KAkodu AK, Email: aakodu@unilag.edu.ng

"Department of physiotherapy, College of Medicine, University of Lagos, Lagos Nigeria 


\section{INTRODUCTION}

There has been a wide geographic and professional variation in the management of low back pain. These variations imply lack of professional consensus on management strategies for low back pain and suggest that some patients may be receiving less than optimal care (1). Despite the availability of clinical practice guidelines, patterns of management with respect to low back pain vary widely and are resistant to change (2). Health care providers often rely on shared beliefs and personal opinions rather than research evidence to make treatment decisions (3). According to Ayanniyi et al (4), up to $35 \%$ of patients with LBP develop chronic problems. This tendency for recurrence and chronicity along with poor response to treatment probably lead to the development of a wide variety of treatment approaches by many health care providers from different professional backgrounds (4,5). At least five hundred randomized controlled trials on the management of LBP have been conducted because of the high incidence, prevalence and reoccurrence rates of LBP (6). Thus, these controlled trials have been summarized into clinical practice guidelines to help clinicians make decisions about the best management for LBP (6).

The Institute of Medicine (IOM) defined clinical practice guidelines as statements that include recommendations intended to optimize patient care which are informed by a systematic review of evidence and an assessment of the beliefs and harms of alternative care options (6). They function to influence clinical decisions making, by presenting the clinician with clear recommendations about what to do in particular situations (6).

Clinical practice guidelines are not selfimplementing. Developing clinical practice guidelines and making them available to health care professionals does not ensure their use. Whilst clinical practice guideline developers may have some responsibility for guideline dissemination, they rarely have responsibility for guideline implementation (7). This unwillingness to follow evidence-based practice recommendations is responsible for what has become known as the know-do-gaps, which is the gap between what is known and what is done in practice (8)

These know-do-gaps are particularly evident in conditions in which treatment options are controversial and no single therapy is universally effective (8). However little seem to be known about the knowledge and use of clinical practice guideline by physiotherapists in Nigeria, despite the wealth of information on the importance of clinical practice guideline in the management of low back pain patients by physiotherapists around the world.

This study is therefore designed to assess the knowledge and adherence to clinical practice guidelines in the management of low back pain amongst physiotherapists in selected Hospitals in Lagos state, Nigeria.

\section{MATERIALS AND METHODS}

Description of study area: The study areas were selected Teaching Hospitals, General Hospitals, Specialist Hospitals and Private Physiotherapy Clinics in Lagos State .

Participants: A total of 154 physiotherapists participated in this study. They were recruited from selected private and public physiotherapy outpatient clinics in Nigeria using the purposive sampling technique.

Study design: Descriptive cross-sectional study.

Sampling technique; A purposive sampling technique was employed in this study

\section{Study instrument}

Questionnaire Design: The initial draft of the questionnaire was adapted from a previous study by Ayanniyi et al. (5). This served as the working document used in a focus group consisting of academics and clinicians to develop the final draft. The questionnaire consisted of 34 openended and close-ended semi-structured questions and was divided into four sections:

Section A: This was used to obtain demographic data of the participants, which included, age, gender, highest educational attainment, University of graduation, year of induction into physiotherapy practice and location of practice.

Section B: This was used to obtain work experience of the participants including years of practice, place of practice/ type of health facility, sub-specialty and cadre in physiotherapy practice.

Section C: Sought information on participants treatment activities.

Section D: This was used to obtain information on the participants' knowledge and adherence to LBPClinical practice guidelines.

Prior to the distribution, the questionnaire was sent to two physiotherapy educators at the 
College of Medicine, University of Lagos, and to two clinician at the Lagos University Teaching Hospital; in order to determine the content validity. Corrections were made according to their inputs.

\section{Administration of the questionnaire/ethical consideration}

Prior to the commencement of the study, ethical approval was sought and obtained from the Health Research and Ethics Committee (approval number: ADM/DCST/HREC/APP/067) of Lagos University Teaching Hospital (LUTH), Idi-Araba Lagos before embarking on the research. The purpose of the research was explained to the respondents and their consents were sought before the commencement of the study. Copies of the questionnaire were distributed to practicing physiotherapists in all teaching University Hospitals and other selected Hospitals, these included General Hospitals, Specialist Hospital, Military Hospitals, Sport Centers, and Private Physiotherapy Clinics. The questionnaires were distributed on a one-on-one basis to practicing physiotherapists located in Lagos State and by email to those who find it more convenient. The aims of the study were clearly explained to all the participants in a cover note on each of the questionnaires in order to seek their consent.

Measurement of outcome variables: This was assessed using the section $\mathrm{D}$ of the questionnaire that asked questions on the knowledge and adherence to low back pain clinical practice guideline

Data Analysis: The data was analyzed using the Statistical Package for Social Sciences (SPSS) version 20. Descriptive statistics of frequencies and percentages were calculated. The results were represented using tables, pie charts and bar charts. Chi-square analysis was used to determine significant associations between the characteristics of the physiotherapists and their knowledge and adherence to LBP clinical practice guidelines. The level of significance was set at $\mathrm{p} \leq 0.05$.

\section{RESULTS}

\section{Socio demographic of respondents}

A total of 154 questionnaires were distributed to various physiotherapy outpatient clinics in Lagos State and 106 copies were returned and valid for analysis giving a response rate of $68.8 \%$. The age range of respondents was between 20 and 60 years, with the majority, $48(45.30 \%)$ between ages of 31-40 years. Majority $63(59.40 \%)$ of the respondents were males while $43(40.60 \%)$ were females. The highest, 51 (48.10) educational attainment for most of the respondents is Master's degree while $16(15.10 \%)$ had doctor of philosophy degree (Ph.D).

Chi square analysis showed that there was no statistically significant association (= 1.42, $\mathrm{p}=0.70$ ) between age of respondent and knowledge of LBP clinical practice guidelines; however, the younger the respondents are, the more knowledgeable they are about the clinical practice guidelines. There was also no statistically significant association $(=5.64, \mathrm{p}=$ 0.13 ) between age of the respondent and adherence to LBP clinical practice guideline (Table 1).However, Chi square analysis showed a statistically significant association $(=11.20 ; \mathrm{p}=$ 0.00 ) between highest level of educational attainment and knowledge of LBP clinical practice guideline and no statistically significant association $(=4.89 ; \mathrm{p}=0.09)$ between highest level of educational attainment and adherence to LBP clinical practice guideline (Table 2).

\section{Work Experiemce}

Concerning years of working as a physiotherapist, majority, 37 (34.90\%) of the respondents have worked for 6-10 years, 26 (24.50\%) had 2 to 5 years of work experience, 23 (21.70\%) had 11 to 15 years of work experience, $15(14.20 \%)$ had 16 to 20 years of work experience, $4(3.80 \%)$ had 21-25 years of work experience and $1(0.90 \%)$ had work experience of 21 to 30 years (figure1). However, Table 3 showed that there was no statistically significant association $(=8.96, p=0.11)$ between years of working experience and knowledge of LBP clinical practice guidelines but there was a statistically significant association $(=11.06, p=$ 0.05 ) between years of working experience and adherence to LBP clinical practice guidelines (Table 3).

Thirty-seven $(34.90 \%)$ of the respondents worked in teaching hospitals, 9 $(8.50 \%)$ worked at private physiotherapy clinics, $8(7.50 \%)$ worked in military hospitals, $1(0.90 \%)$ worked in Sport Center, 18 (17.00\%) worked in specialist hospital, $24(22.60 \%)$ worked in general hospitals, 2 (1.90\%) worked as Domiciliary, 4 (3.80\%) were Academicians and 3 $(2.80 \%)$ worked in Federal Medical Centers. Chi 
square analysis showed that there was a statistically significant association $(=15.52$. $\mathrm{p}=0.05$ ) between setting of practice and knowledge of LBP clinical practice guideline, and between setting of practice and adherence to LBP clinical practice guidelines $(=19.63, \mathrm{p}=$ 0.01) (Table 4).

Regarding the areas of specialization of the respondents, $21(19.80 \%)$ specialized in Neurology, 7 (6.60\%) specialized in Paediatrics, $2(1.90 \%)$ specialized in Ergonomics, 63 $(59.40 \%)$ specialized in Orthopaedics, 11 $(10.40 \%)$ specialized in Cardiopulmonary, 2 $(1.80 \%)$ specialized in Sports. Chi square analysis showed a statistically significant association $(=15.16, p=0.02)$ between area of specialization and knowledge of LBP clinical practice guidelines, it also showed a significant association $(=17.50, p=0.01)$ between area of specialization and adherence to LBP clinical practice guidelines (Table 5). Eighty-three $(78.30 \%)$ of the respondents have undergone an additional training on the management of low back pain while $23(21.70 \%)$ have not.

Chi-square analysis revealed that there was a statistically significant association $(=5.56$, $\mathrm{p}=0.02$ ) between post qualification training and knowledge of LBP clinical practice guidelines but no statistically significant association $(=2.92$, $\mathrm{p}=0.09$ ) between post qualification training and adherence to LBP clinical practice guidelines (Table 6).

Treatment Activities: All of the respondents 106 $(100.00 \%)$ manage patients with low back pain, with most of the respondents $67(64.20 \%)$ treating between 1 to 10 patients per week, 27 (25.40\%) treating between 11 to 20 per week, 9 $(8.50 \%)$ treating 21 to 30 patients per week and 2 $(1.90 \%)$ treating 31 and above (Figure2).

Chi-square analysis showed no statistically significant association $\left(\mathrm{x}^{2}=2.96, \mathrm{p}=0.23\right)$ between physiotherapists who manage LBP and knowledge of LBP clinical practice guidelines nor between physiotherapists who manage LBP and adherence to LBP clinical practice guidelines $(=2.08, \mathrm{p}=0.35)($ Table 7$)$.

The major factor for the choice of modalities/techniques such as electrotherapy modalities / manipulative, massage and mobilization technique etc chosen by the respondents in their management of low back pain is skill $82(77.40 \%)$, followed by diagnosis $81(76.40 \%)$, then availability with $74(69.80 \%)$, while $16(15.10 \%)$ use number of patients to determine their choice of modality .

Sixty-six $(62.90 \%)$ respondent spend between 41-60 minutes treating their patients with LBP for the initial visit, 13 (12.40\%) respondent spend between 61-80 minutes for the initial visit, $10(9.50 \%)$ respondents spend between 20-40 minutes treating their patients with LBP for the initial visit, $6(5.70 \%)$ respondents spend between 81 to 100 minutes treating their patients with LBP for the initial visit, $6(5.70 \%)$ spend between 101 to 120 minutes treating their patients with LBP for the initial visit and $5(3.80 \%)$ respondents spend about 120 minutes and above treating patients with LBPfor the final visit.

However, for the follow-up visits in the management of low back pain, $7(6.70 \%)$ respondents spend 20 minutes and below, 53 $(50.40 \%)$ respondents spend between 21-40 minutes, 39 (37.20\%) respondents spend 41-60 minutes, $1(0.90 \%)$ respondents spend $61-80$ minutes, $4(3.80 \%)$ respondents spend $81-100$ minutes and $2(1.00 \%)$ respondents spend 101120 minutes. Majority $72(70.60 \%)$ of the respondents use between 1-10 treatment sessions for the management of patients with LBP, 27 (26.50\%) respondents use between 11-20 treatment sessions and $7(2.90 \%)$ respondents use between 21-30 treatment sessions for the management of LBP . Seventy-six $(71.70 \%)$ respondents gave prognosis as the major factor for their choice of sessions, 66 (62.30\%) respondents gave diagnosis as a factor for their choice of treatment session and $24(21.70 \%)$ gave availability of modalities as a factor for their choice of treatment sessions.

Majority 98 (92.50\%) of the respondents used Exercise therapy in the management of LBP, $97(91.50 \%)$ used Electrotherapy modalities, 76 $(71.70 \%)$ used Manipulative therapy, 92 $(86.80 \%)$ used Patient education and counselling, $81(76.40 \%)$ used Mobilization techniques, 49 (46.20\%) used Traction, $97(91.50 \%)$ used Massage therapy, $7 \quad(6.60 \%)$ used Thermotherapy, 5 (4.70\%) used Cryotherapy and $2(1.90 \%)$ used Rest (Table 8).

The major factor for the choice of modalities/techniques chosen by the respondents in their management of low back pain is skill 82 (77.40\%), followed by diagnosis $81(76.40 \%)$, then availability with $74(69.80 \%)$, while 16 $(15.10 \%)$ use number of patients to determine their choice of modality. 


\section{Knowledge and Adherence to LBP Clinical Practise Guidelines}

Forty-seven $(44.30 \%)$ of the respondents are aware of the LBP clinical practice guidelines while $59(55.70 \%)$ are not aware (Table 8). Majority $76(71.70 \%)$ are not knowledgeable while $30(28.30 \%)$ of the respondents were knowledgeable. However, $23(21.70 \%)$ of the respondents adhere to the LBP clinical practice guidelines while $83(78.3 \%)$ of the respondents do not.

Ninety $(84.90 \%)$ of the respondents are knowledgeable that there is no clinical practice guideline for LBP in Nigeria while $16(15.10 \%)$ thinks there is. Most of the respondent 79 (74.50\%) think there is need for a low back pain clinical practice guideline for the practicing physiotherapists in Nigeria, while 23 (21.70\%) are not sure if there is a need for guidelines.

The response given by majority $15(14.20 \%)$ for the reason low back pain clinical practice guideline is needed in Nigeria is for standardization of practice. One $(0.90 \%)$ respondent out of those that said there is no need for clinical practice guidelines gave a reason that we naturally conform to evidence based practice in our clinical setting while another says that there will be ineffective dissemination even after the LBP clinical practice guideline has been made available.

Four $(3.8 \%)$ of the respondent out the respondent that are aware of clinical practice guideline mentioned American Pain Society joint clinical practical guideline for diagnosis and treatment of LBP, 3(2.8\%) mentioned Australian clinical practice guideline, $0.9 \%$ Philadelphia panel evidence based clinical practice guideline on selected rehabilitation interventions for LBP, 4(3.8\%) mentioned The American College of Physicians, 3(2.8\%) mentioned Evidence based management of LBP, 8(7.5\%) mentioned Mckenzie while 2(1.9\%) mentioned Nwugarian technique.

\section{DISCUSSION}

The overall aim of the study was to determine the knowledge and adherence to LBP clinical practice guidelines amongst physiotherapists in selected Hospitals in Lagos state and also to determine if the physiotherapists' characteristics (such as age, highest educational attainment, number of years of experience, setting of practice and area of specialization) influence the physiotherapists knowledge and adherence to LBPclinical practice guidelines.
It was evident from this study that physiotherapists use variety of treatment modalities with exercise therapy being the most popular approach for managing patients with LBP. Electrotherapy modalities and Massage were among the most common treatment preferences followed by patient's education and counselling. This finding is in agreement with the results of the studies of Battie et al. (9), and Ayanniyi et al. (4) which indicated exercise therapy as well as patient's education as most popular treatment method used for the management of LBP.

The findings from this study revealed that skills, diagnosis and availability of treatment modalities and techniques were the most common factors that influenced respondents' choice of treatment modalities. This implies that the knowledge of the respondents and the acquisition of additional training may have an influence on choice of treatment modalities.

The findings of this study revealed that majority of the respondents discharge their patients after about 10 treatment sessions. This is in agreement with the findings of Swinkels et al. (10) and Odebiyi et al. (5), who reported that the mean number of physical therapy treatment sessions in patients referred with non-specific LBP is 9.9 sessions.

In this survey, more than $98 \%$ of the respondents did not use bed rest in the management of LBP. This finding is in agreement with studies by Koes et al. (11) and Goertz etal. (12) as there is now a broad consensus that bed rest should be discouraged as a treatment for low back pain. A gradual return to normal activities is more effective and leads to more rapid improvement with less chronic disability. But then, if bed rest is to be recommended in cases of severe pain, it should not be for more than 2 days $(12,11)$. These findings are in agreement with the guideline recommendations.

The European clinical practice guidelines for chronic non-specific LBP (13), discourage the use of modalities such as TENS, ultrasound, and microwave diathermy, which possess uncertain effectiveness for managing acute LBP, however, about $91.5 \%$ of the respondents use electrotherapy modalities in managing patients with LBP. This is in agreement with findings from results of the study of $\mathrm{Li}$ and Bombardier (14) who carried out a survey on Canadian physical therapists on how they managed acute/sub-acute low back pain (LPB), using clinical vignettes. Despite the clinical 
practice guidelines on LBP management, it was found that more than one third of physical therapists still use electrotherapeutic modalities of uncertain effectiveness (14).

The results of this study suggest that there is a gap between knowledge and action that needs to be bridged if efforts to maximize guideline compliance are to succeed. This finding is in line with the practice of physiotherapists as reported by Di lorio (2) and Pablos-mendez and Shademani (8).

It was reported that an average population of the respondents are aware of the LBP clinical practice guidelines When asked to mention the ones they know, some of the respondents mentioned some of the available clinical practice guidelines such as Philadelphia panel evidence based clinical practice guideline on selected rehabilitation interventions for LBP, The American College of Physicians and American Pain Society joint clinical practice guideline for diagnosis and treatment of LBP, Australian clinical practice guidelines e.t.c. making them knowledgeable while some mentioned treatment protocols such as Mckenzie technique, Nwugarian technique, Evidence -based practice in the management of low back pain making a larger population not knowledgeable. This implies that there is a misconception amongst physiotherapists about the difference between LBP clinical practice guidelines and treatment protocols in the management of LBP.

The finding that there was no significant relationship between age and years of work experience of the respondents and knowledge of clinical practice guideline, suggests that age and years of work experience may not have an influence on the physiotherapists' knowledge of LBP clinical practice guidelines. However, it was observed that the younger the physiotherapists, the more knowledgeable they are about LBP clinical practice guidelines.

The findings from this study revealed that there is an association between setting of practice and knowledge of clinical practice guidelines, which may suggest that the setting of practice of the physiotherapist may influence his/her knowledge of LBP clinical practice guidelines. Respondents working in specialist Hospitals were the most knowledgeable and this may be because they major mostly in orthopaedic conditions.

The finding that there was a significant association between area of specialization of respondents and knowledge of LBP clinical practice guidelines suggests that the area of specialization of the physiotherapist may influence his/her knowledge about LBP clinical practice guidelines. It was observed that those who specialize in orthopaedic conditions are more knowledgeable about LBP clinical practice guidelines.

It was interesting to find out that there was no relationship between respondent's involvement in the management of LBP and knowledge of LBP clinical practice guidelines as it would have been assumed that those involved in managing LBP would have a wider knowledge base about LBP management and evidence-based practice. Since the acquisition of post qualification training by the respondents has being reported to influence the knowledge of clinical practice guidelines. This implies that physiotherapists with additional qualifications, particularly in the management of LBP, are knowledgeable about LBP clinical practice guidelines.

Out of the low population of physiotherapist who are knowledgeable about LBP clinical practice guideline, only a small percentage $(21.7 \%)$ adhere to this guidelines in their current practice of managing LBP. This implies that along with implementation and availability of these guidelines, knowledge of the practice guidelines does not ensure the adherence of physiotherapists to clinical practice guidelines in the management of LBP. This finding concurs with that of Shekelle et al. (7) who reported that guidelines are not self-implementing. Whilst guideline developers may have some responsibility for guideline dissemination, they rarely have responsibility for guideline implementation (7).

This study revealed that there was a significant association between setting of practice and area of specialization of respondents and usage of LBP clinical practice guidelines. Similarly, there was an association between post qualification training and usage of LBP clinical practice guidelines. This finding implies that the knowledge base of the physiotherapist influences the usage of these guidelines as those who work in specialist Hospitals and specialize in Orthopaedics use the guidelines more often.

Most of the respondents in this study were aware that there are no available guidelines in Nigeria. However, a low percentage $(1.9 \%)$ believes the Nwugarian technique is a LBP clinical practice guideline. This finding emphasizes the previous point that some of the respondents do not know the difference between 
clinical practice guidelines and treatment protocols for LBP.

Majority of the respondents opined that there is a need to develop LBP clinical practice guidelines in Nigeria. This according to them will standardize practice, reduce variation in treatment protocols, increase the use of evidencebased physiotherapy and promote better and more effective outcome in the management of LBP patients. This finding conform with the report of the study of Odebiyi et al. (5) who also found out that there is a need to develop LBP clinical practice guidelines in Nigeria so as to standardize practice, encourage proper diagnosis and the use of evidence-based physiotherapy management. It has been reported that the main benefit of clinical practice guidelines is to improve the quality of care of patients (1), as clinical practice guidelines are usually aimed at increasing the quality of health care provided, it has been found to decrease the number of visits of patients with LBP in the Netherlands physiotherapist clinics (15).

\section{CONCLUSION}

A know-do gap clearly exists among physiotherapists with respect to the treatment of LBP, particularly in the advice given to patients regarding active treatments and use of electrotherapeutic modalities. Guidelines are often used to establish standards of care and provide a benchmark for evidence-based practice, but the results of the present report demonstrated that their directives are not always adhered to.

Educational attainment, setting of practice, area of specialization and post qualification training of the physiotherapists all influences the knowledge of LBP clinical practice guidelines while age, years of work experience and involvement in management of LBP did not have any significant influence on their knowledge of LBP clinical practice guidelines.

However, years of work experience, setting of practice and area of specialization of the physiotherapists influences their adherence to LBP clinical practice guidelines while age, educational attainment, post qualification training and involvement in the management of LBP did not influence the physiotherapists' adherence to LBP clinical practice guidelines.

There was a broad consensus that a clinical practice guideline is needed for the proper management of patients with LBP in Nigeria.

It is therefore recommended that a LBP clinical practice guideline should be developed in Nigeria as most of the physiotherapists advocated for it. Although it is not possible to dictate or change the personal opinion of a health care practitioner, education in the form of continuous professional development (CPDs) and experience may eventually erode obstructive attitudes and beliefs that could adversely affect patient care. However, knowledge of clinical practice guidelines, are insufficient for improving practice; active, deliberate, and evidence-based implementation strategies are often required. Physiotherapists and health care practitioners in general need to be aware of recent changes in the environment and thus, be able to standardize and rationalize the management of low back pain according to adequate standards of scientific quality.

Conflicts of interest: None declared.

Acknowledgement: The authors acknowledge the contribution of all the subjects that participated in this study.

\section{REFERENCES}

1. Woolf SH, Grol R, Hutchinson A. Clinical guidelines: potential benefits, limitations and harms of clinical guidelines. BioMedicalJournal, 1999, 318:527-530.

2. Di Lorio D, Henley E, Andrea D. A survey of primary care physician practice patterns and adherence to acute low back problem guidelines.Archives of Family medicine, 2000, 9: 1015-1021

3. Spring B, Pagoto S, Kautmann PG. Invitation to a dialogue between researchers and clinicians about evidence-based behavioural medicine. Annals of behavioural medicine, 2005, 30: 125-37.

4. Ayanniyi O, Lasisi OT, Adegoke BOA, Oniorisan MO. Management of LBP: attitudes and treatment preferences of physiotherapists. AfricanJournalofBiomedicalresearch, 2007, 10:41-49.

5. Odebiyi DO, Aweto HA, Igbari TO, Tella BA. Factors influencing number of physiotherapy treatment sessions for patients with LBP. African Journal of Physiotherapy and Rehabilitation Sciences, 2012. 4: 23-28.

6. Ladeira CE. Evidence based practice guidelines for management of low back pain: Physical Therapy Intervention. Brazillian journal of physical therapy, 2011, 15(3): 14133555 . 
7. Shekelle P, Woolf S, Grimshaw JM, Schuemann HJ, Eccles MP. Developing clinical practice guidelines: reviewing, reporting, and publishing guidelines: updating the guidelines and the emerging issues of enhancing guideline implementability and accounting for co-morbid conditions in guideline development. Implementation Science, 2012, 7: 62.

8. Pablos-mendez A, Shademani R. Knowledge translation in global health. $\mathrm{J}$ continuing Education Health Profession, 2006, 26:81-6.

9. Battié MC, Ciol MA, Wheeler KJ, Cherkin DC, Dunn R. Management of LBP: Attitudes and treatment preferences of physiotherapists. Physical Therapy, 1994, 74(3): 219-228.

10. Swinkles CS, Wimmers RH, Groenewegen PP, Van debosch JH, Dekker J, Ende HM. What factors explain the number of physical therapy sessions in patients referred with low back pain? Spine, 2005, 4: 212-222.

11. Koes BW, Van Tulder MW, Lin CW, McAuley J, Macedo LG, Maher C. An updated overview of clinical guidelines for the management of LBP in primary care. European Spine Journal 2010, 19: 2075-2094.

12. Goertz M, Thorson D, Bonsell J, Bonte B, Campbell R,Haake B, Johnson K, Kramer C, Mueller B, Peterson S, Setterlund L, Timming R. Adult acute and sub-acute LBP. Institution for clinical systems approvement, 2012. A v a i 1 a b 1 e a $\mathrm{t}$ www.icsi.org/asset/bjvqrj/LBP.pdf.Accessed at 24/08/2015.

13. Van Tulder MW, Tuut M, Pennick V, Bombardier C, Assendelft WJ. Quality of primary care guidelines for acute low back pain. Spine, 2004, 29(17): 357-62.

14. Li LC, Bombardier C. Physical Therapy Management of Low Back Pain: An Exploratory Survey of Therapist Approaches. Physical Therapy, 2001, 81(4): 1018-1028.

15. Groenengijk JJ, Swinkles S, Bakker D, Dejjer J, Van den Ende CJ. Physical therapy management of LBP has changed. Health Policy 2007, 80: 492-499 


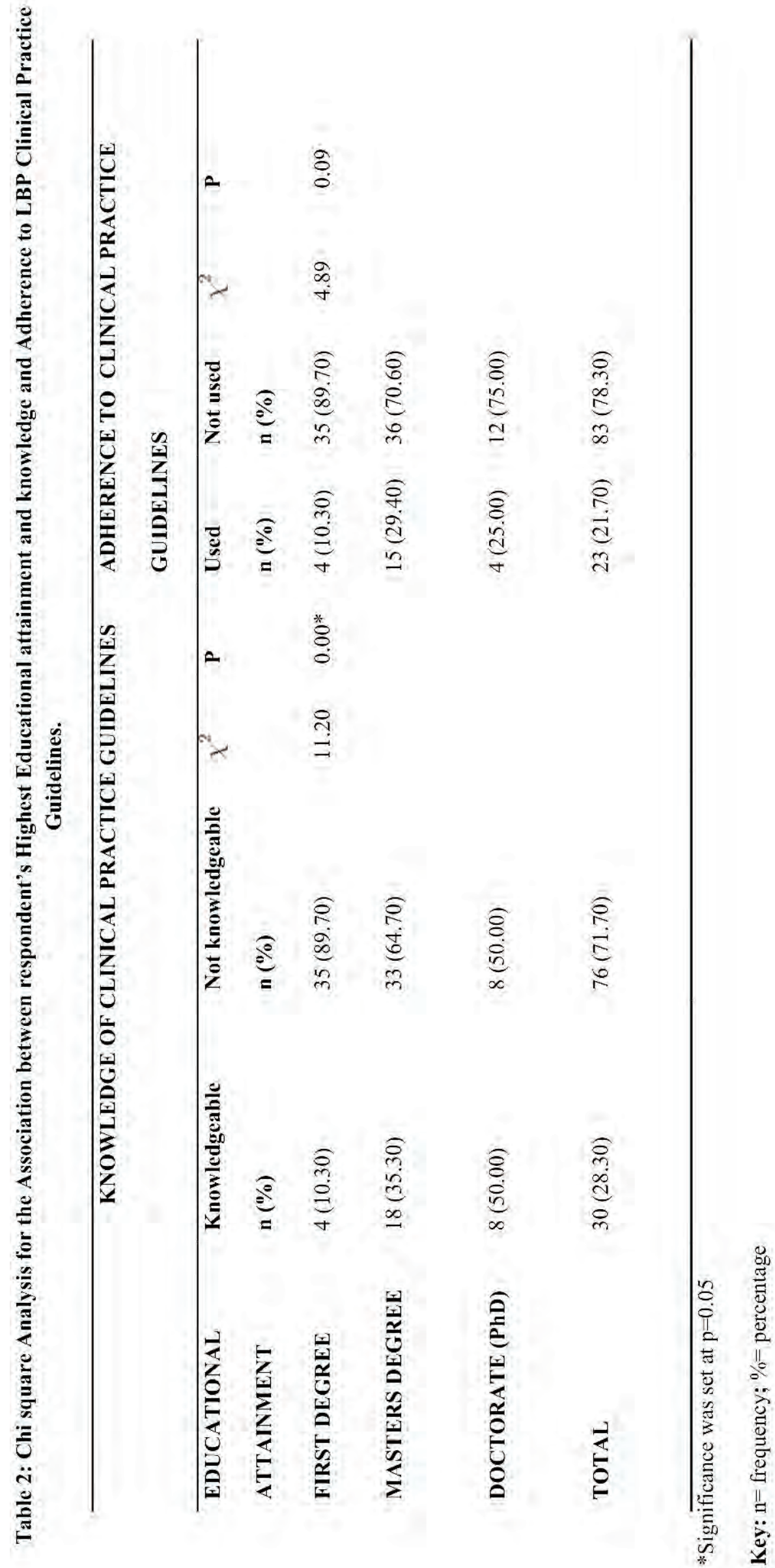




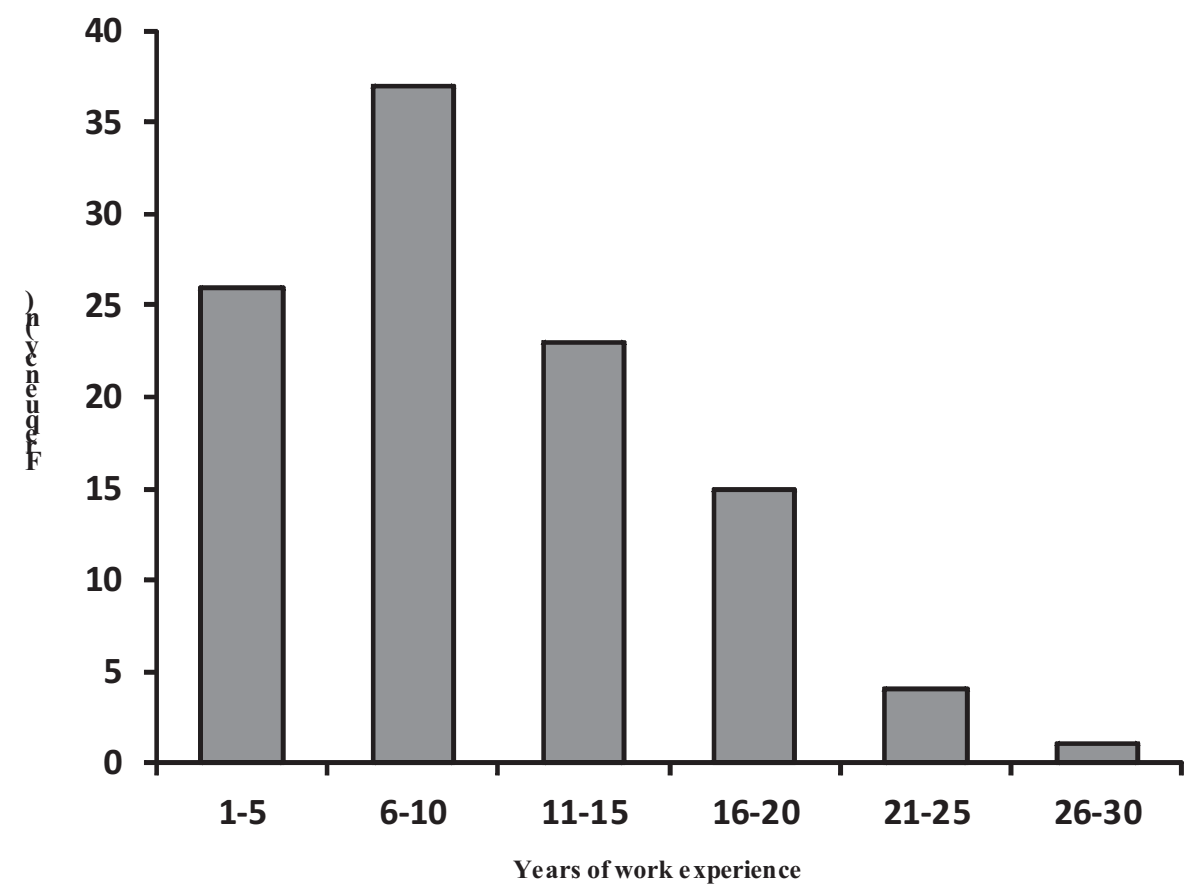

Figure 1: Frequency Distribution of Respondent's Years of Work Experience 


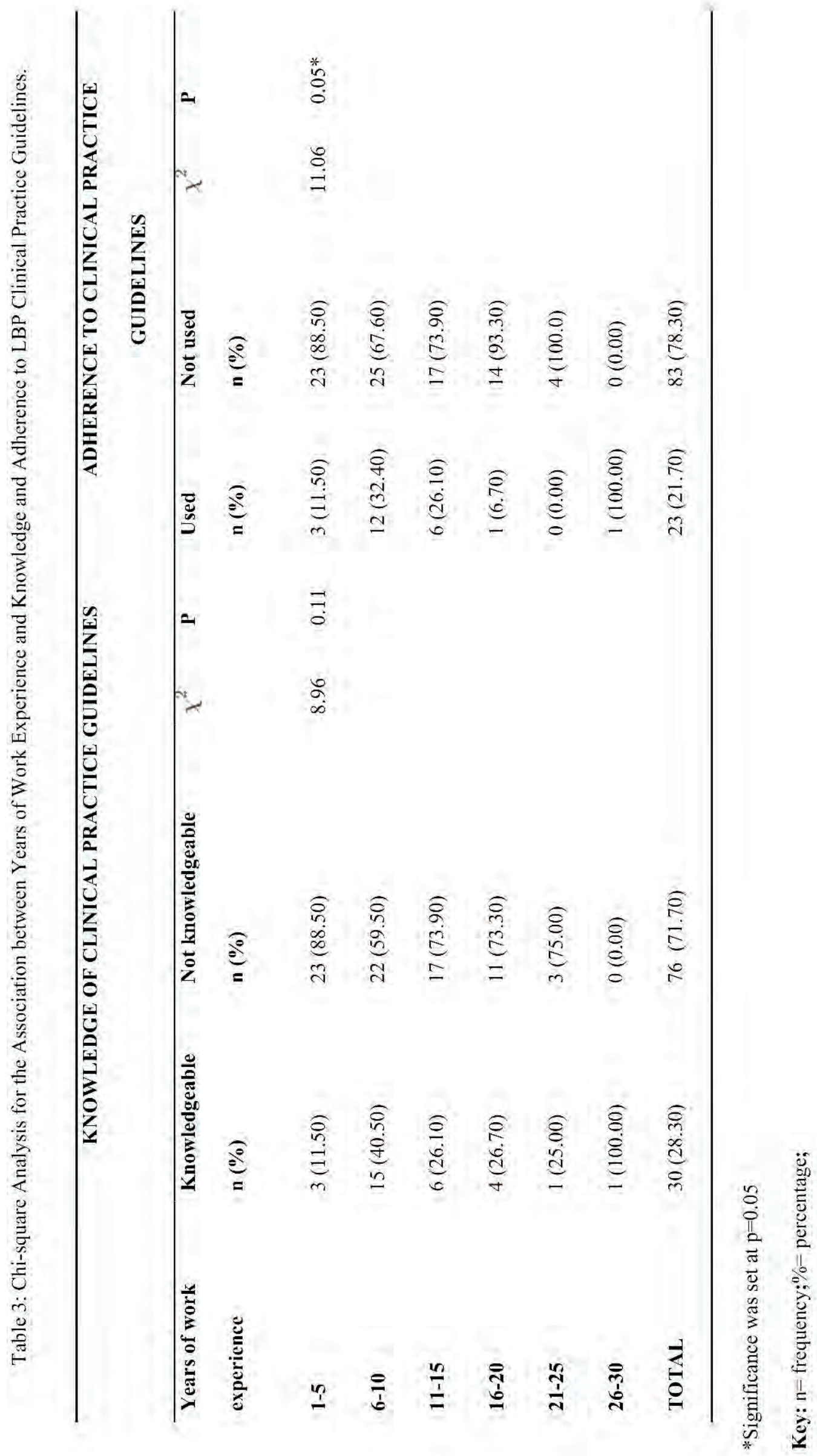

Res. J. of Health Sci. Vol 4(3), July/September 2016 


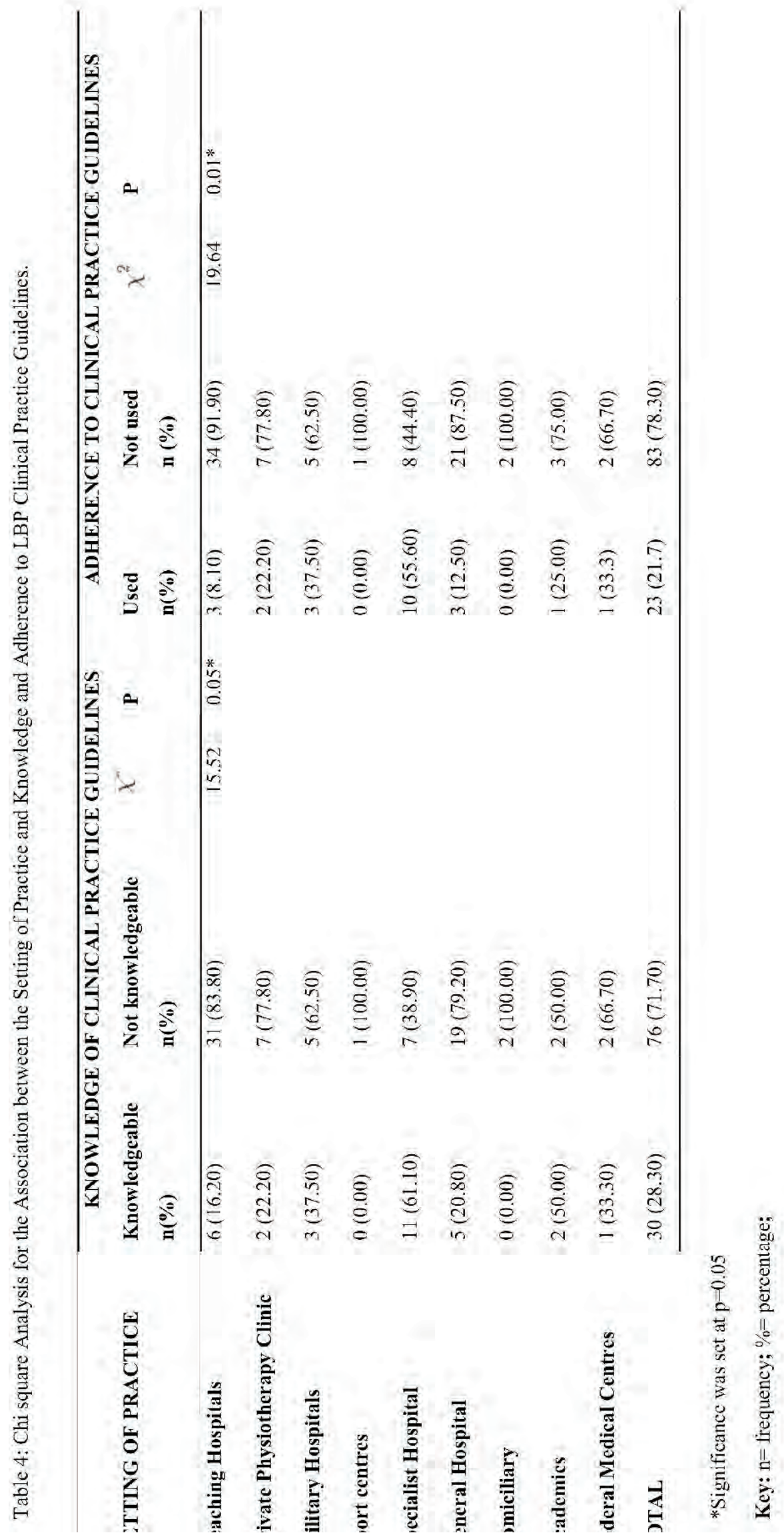




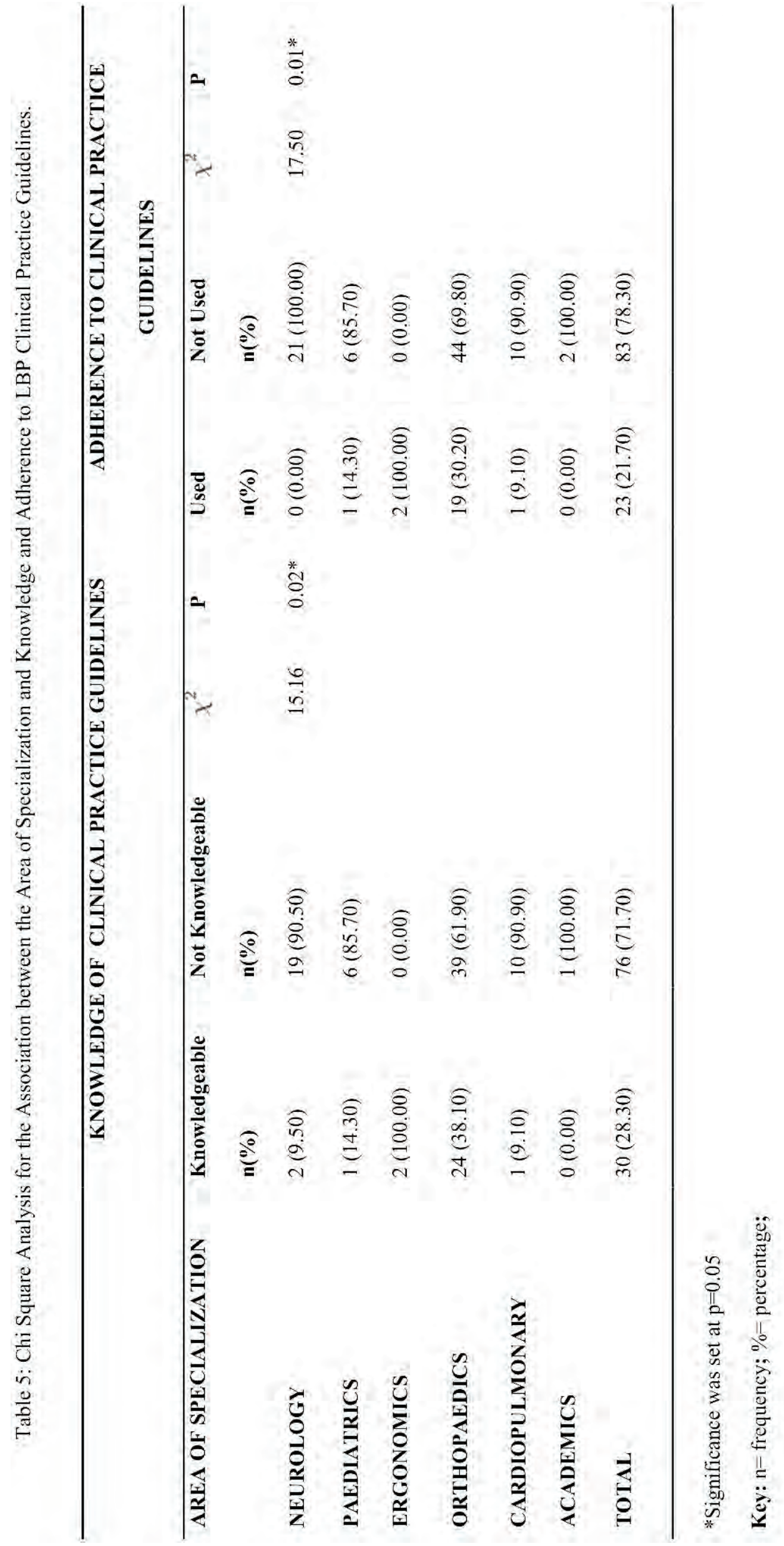




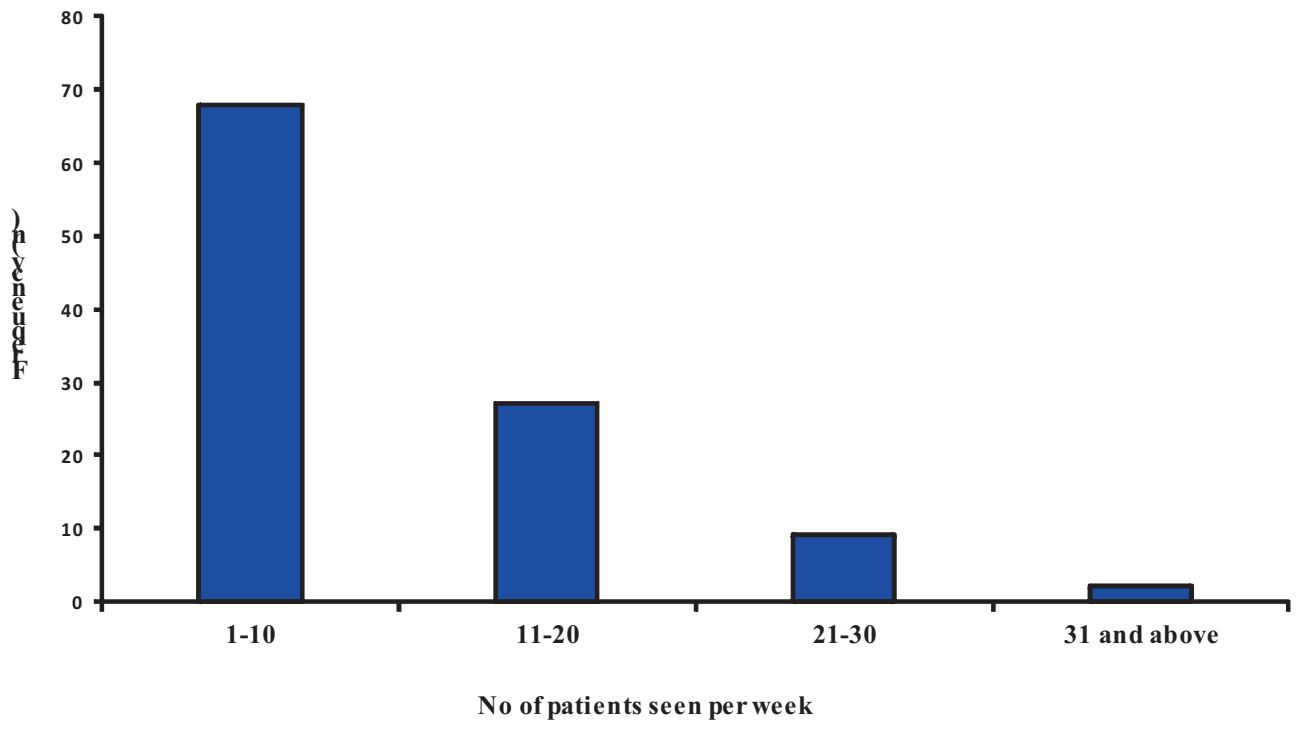

Figure 2: Frequency distribution of patients seen by respondents per week. 


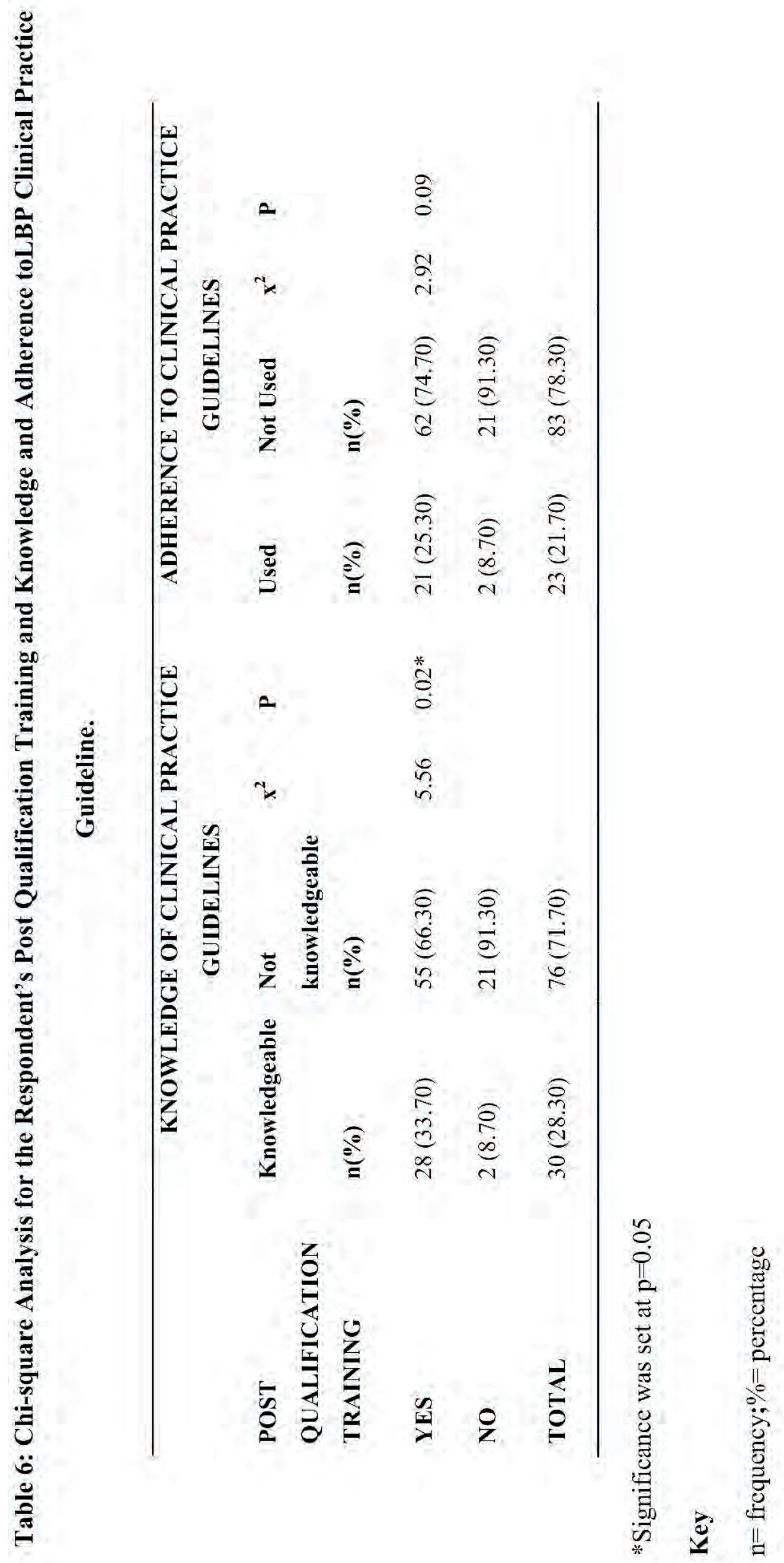


Table 7: Frequency Distribution of the Type of Modalities/Techniques used by the respondent.

\begin{tabular}{lll}
\hline & $\begin{array}{l}\text { FREQUENCY } \\
(\mathbf{n})\end{array}$ & $\begin{array}{l}\text { PERCENTAGE } \\
(\%)\end{array}$ \\
TYPE OF MODALTIES/TECHNIQUES USED & & \\
Electrotherapy modalties & 97 & 91.50 \\
Manipulative therapy & 76 & 71.70 \\
Patient education/counselling & 92 & 86.80 \\
Mobilization techniques & 81 & 76.40 \\
Traction & 49 & 46.20 \\
Massage therapy & 97 & 91.50 \\
Exercise therapy & 98 & 92.50 \\
Thermotherapy & 7 & 6.60 \\
Cryotherapy & 5 & 4.70 \\
Rest & 2 & 1.90 \\
\end{tabular}

Table 8: Frequency Distribution of Respondent's Awareness, Knowledge and Adherence to LBP Clinical Practice Guideline

$\begin{array}{ll}\begin{array}{l}\text { FREQUENCY } \\ \text { (n) }\end{array} & \begin{array}{l}\text { PERCEN } \\ (\%)\end{array} \\ & \\ & \\ & \\ 47 & 44.30 \\ 59 & 55.70 \\ 106 & 100.00\end{array}$

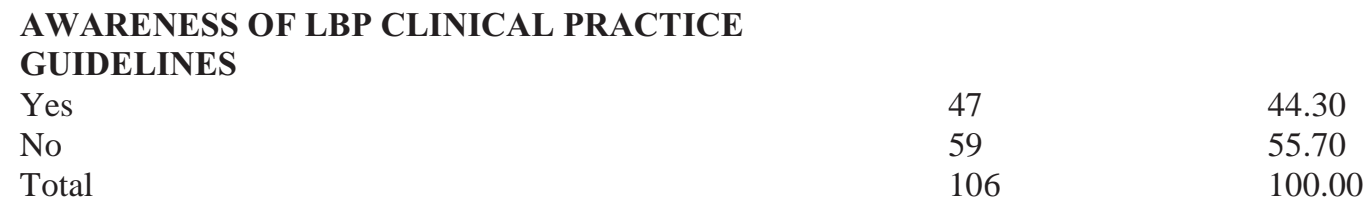

KNOWLEDGE OF LBP CLINICAL PRACTICE

\section{GUIDELINE}

Knowledgeable

30

28.30

Not knowledgeable

76

71.70

Total

106

100.00

\section{ADHERENCE TO LBP CLINICAL PRACTICE}

\section{GUIDELINE}

Adhered to

Not adhered to

Total

$\begin{array}{ll}23 & 21.70 \\ 83 & 78.30 \\ 106 & 100.00\end{array}$




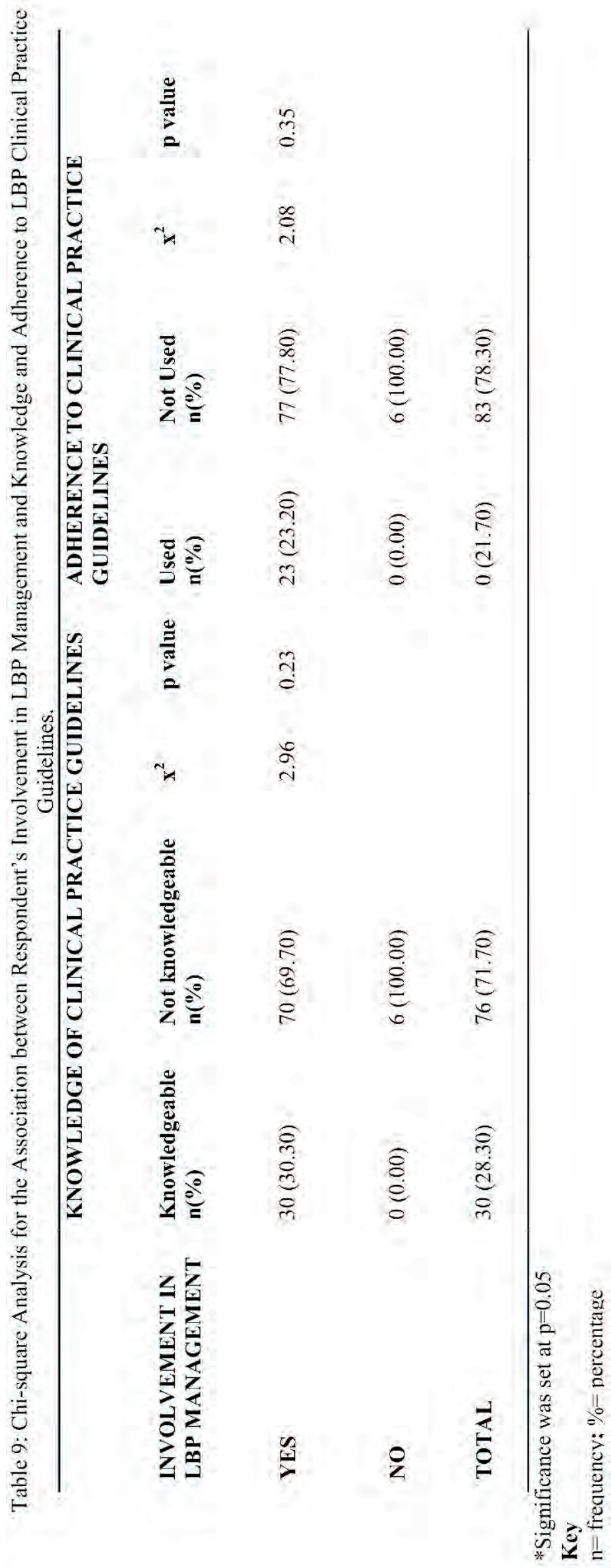

